\title{
Overview of tobacco use transitions for population health
}

\author{
Andrew Hyland, ${ }^{1}$ Karin A Kasza (10 , ${ }^{1}$ Nicolette Borek, ${ }^{2}$ Heather L Kimmel 다, ${ }^{3}$ \\ Kristie A Taylor 이, ${ }^{4}$ Wilson M Compton, ${ }^{3}$ Hannah Day, ${ }^{2}$ Elisabeth A Donaldson, ${ }^{2}$

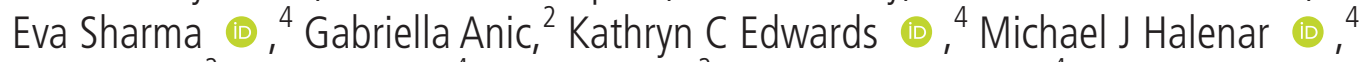 \\ Lynn C Hull, ${ }^{2}$ Wendy Kissin, ${ }^{4}$ Jean Limpert, ${ }^{2}$ Elizabeth L Seaman, ${ }^{4}$ \\ Maansi Bansal-Travers (10), Lisa D Gardner (1) , ${ }^{2}$ Hoda T Hammad, ${ }^{2}$ \\ Cassandra A Stanton (1) ${ }^{4,5}$
}

${ }^{1}$ Department of Health Behavior, Roswell Park Comprehensive Cancer Center, Buffalo, NY, USA ${ }^{2}$ Office of Science, Center for Tobacco Products, Food and Drug Administration, Silver Spring, MD, USA

${ }^{3}$ National Institute on Drug Abuse, National Institutes of Health, Bethesda, MD, USA ${ }^{4}$ Behavioral Health and Health Policy Practice, Westat, Rockville, MD, USA

${ }^{5}$ Department of Oncology, Georgetown University Medical Center, Washington, DC, USA

\section{Correspondence to}

Dr Andrew Hyland, Department of Health Behavior, Roswell Park Comprehensive Cancer Center, Buffalo, NY 14263, USA; andrew.hyland@roswellpark.org

Received 5 September 2019 Revised 14 January 2020 Accepted 15 January 2020

\section{ABSTRACT}

The Family Smoking Prevention and Tobacco Control Act provided the US Food and Drug Administration authority to regulate tobacco products using a population health standard. Models have been developed to estimate the population health impacts of tobacco initiation, cessation and relapse transitions. Models should be informed by high-quality, longitudinal data to estimate these constructs. Simulation studies have generated data to predict the impact of various tobacco control interventions, including the influence of regulations on tobacco use behaviours and health. The purpose of this paper is to provide a high-level conceptual overview for understanding tobacco transition behaviours and correlates of these behaviours using data from the Population Assessment of Tobacco and Health (PATH) Study, a US nationally representative longitudinal tobacco study of about 46000 persons aged 12+ years. The papers that follow in this journal issue build and expand on this conceptual overview using data from the first three waves of the PATH Study. These papers describe use patterns of different tobacco products and their correlates, and can serve as foundations for more in-depth papers that will help the research community better understand the population health impacts and drivers of different tobacco use patterns.

\section{INTRODUCTION}

Cigarettes remain the predominant type of tobacco product used in most countries, although the scope of tobacco products available today has expanded and includes non-cigarette tobacco products such as electronic nicotine delivery systems (ENDS), hookah (waterpipe), cigars and smokeless tobacco products. $^{1-9}$ In India and Bangladesh, smokeless tobacco use exceeds cigarette use, and hookah use is particularly high in Vietnam, Egypt, Turkey, the Russian Federation and Ukraine. ${ }^{1}$ However, cigarette smoking still accounts for the overwhelming majority of tobacco-related disease and death in the world; ${ }^{10}$ quitting cigarette smoking reduces the risk of cancer, heart disease and other diseases, and reduces the risk of dying from smoking-related causes. $^{1112}$

Countries around the world have taken steps to address the public health harms caused by tobacco products. Most countries have ratified the WHO
Framework Convention on Tobacco Control (WHO FCTC), a treaty that provides comprehensive provisions to nations to adopt policies to address tobacco use. ${ }^{13}$ In the USA, the 2009 Family Smoking Prevention and Tobacco Control Act provided the US Food and Drug Administration (FDA) authority to regulate the manufacturing, marketing and distribution of tobacco products using a standard based on population health, ${ }^{14}$ unlike the 'safe and effective' standard for most other drugs and medical devices regulated by FDA. A population health standard includes health impacts on tobacco product users, and on never users and former users to understand impacts on the population as a whole. For example, FDA has sought input on the potential public health benefits and any possible adverse effects of lowering nicotine in cigarettes, ${ }^{15}$ and FDA would need to consider scientific evidence to assess whether this action meets the population health standard.

Simulation studies have generated data to predict the impact of various tobacco control policies on tobacco use behaviours and health. ${ }^{16-18}$ Any model or assessment of the scientific evidence for regulating according to a population health standard relies on understanding the scope of tobacco use in the population and how tobacco use changes over time, including trajectories within tobacco product type and movement between product types. Previous studies have considered two-product models to estimate the health impacts of tobacco initiation, cessation and relapse ${ }^{19}$ often using cigarettes and ENDS ${ }^{20} 21$ to assess product transitions. The Population Assessment of Tobacco and Health (PATH) Study offers the opportunity to study numerous transitions between specific products and for overall tobacco use with high-quality longitudinal data in a nationally representative US sample.

This paper provides a brief description of the PATH Study and its role in the tobacco research and policy landscape. Specifically, we outline a high-level conceptual overview to guide thinking about three broad transition behaviours: (1) initiation of tobacco use among non-users; (2) cessation of use among current users; and (3) relapse back to tobacco use among former users. These transition behaviours can apply to several different types of tobacco products ${ }^{4-9}$ and may be influenced by a set of demographic and tobacco use correlates. ${ }^{22-24}$ The papers that follow in this journal issue use this 


\section{Behavioural Transitions}

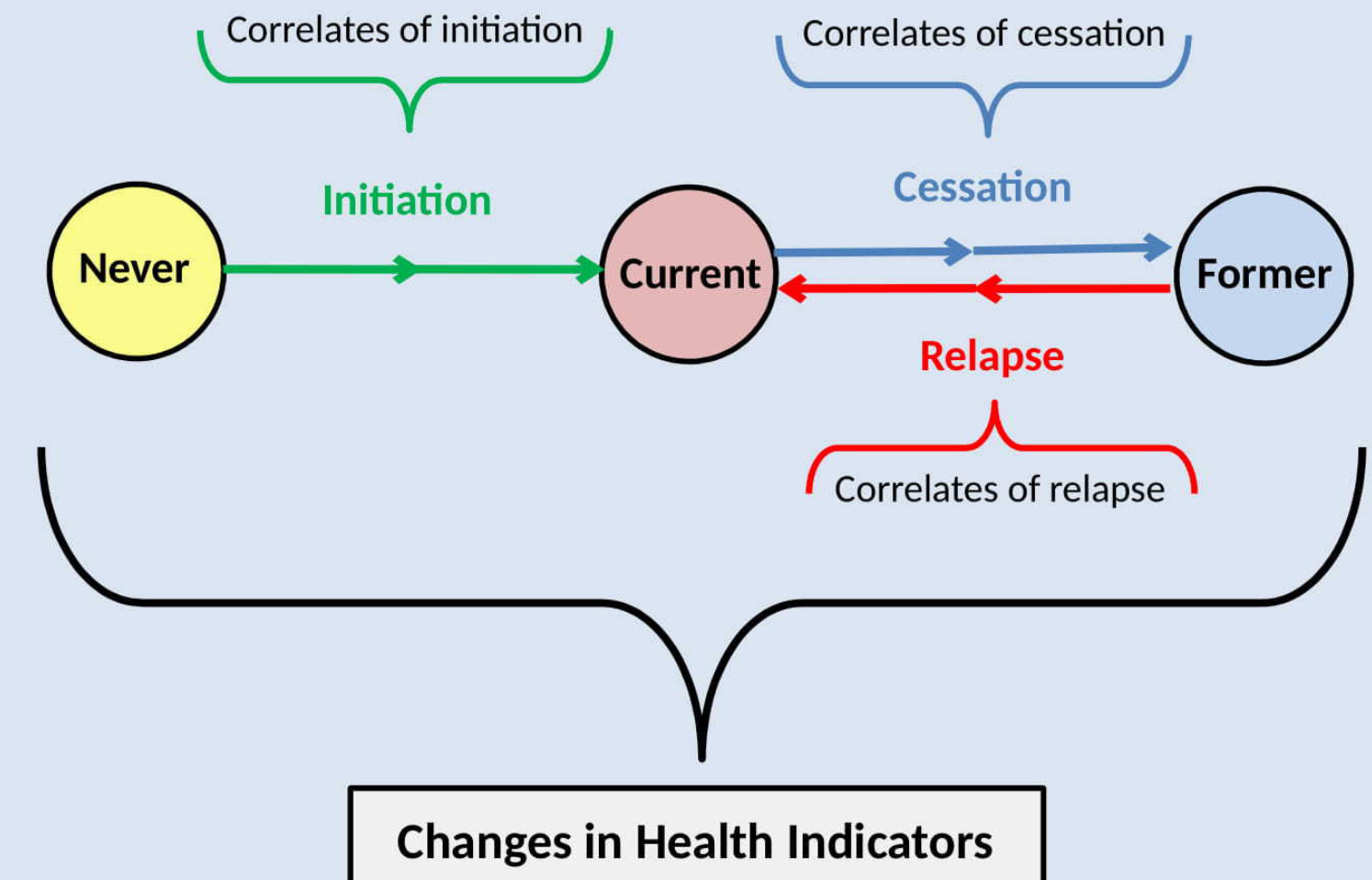

Figure 1 Overview of broad tobacco use transitions for population health. Population-level depiction of three mutually exclusive and exhaustive tobacco user states (ie, never, current, former), three broad behavioural transitions among tobacco user states (ie, initiation, cessation, relapse), each of which is composed of a set of arrows reflecting steps in the process of moving from one user state to another (eg, cessation could include attempting to quit and successful quitting among attempters), correlates of behavioural transitions and how these interact to produce changes in health indicators. Transitions in user states and changes in health indicators are assessed among individuals but reflect population-level health when aggregated together.

overview for different tobacco products among youth 12-17 years of age, young adults 18-24 years of age and adults 25 years of age and older. These papers are a starting point that will serve as a foundation for more in-depth future papers addressing factors and use patterns that are most important for population health.

\section{OVERVIEW OF TOBACCO USE TRANSITIONS FOR POPULATION HEALTH}

Figure 1 depicts three mutually exclusive and exhaustive tobacco user states: never, current and former. User states can pertain to tobacco use in general or specific types of tobacco products. While many different types of behavioural transitions can be examined, the arrows in the figure represent three broad transitions: initiation, which is moving from the never user state to the current user state; cessation, which is moving from the current user state to the former user state; and relapse, which is moving from the former user state to the current user state. Although the figure shows these broad transitions assessed discretely between two time points, in reality they occur continuously, so repeated assessments longitudinally are needed to understand behaviours over time. Initiation, cessation and relapse may repeat over multiple time points to produce longitudinal patterns of tobacco product use/non-use.

In addition to the broad transitions between initiation, cessation and relapse of tobacco use or for one specific product (eg, cigarette smoking cessation), individuals may also switch between product types (eg, cigarettes to ENDS), and transitions can be characterised in several patterns that are illustrated across more than two time points. Five papers in this issue present data on patterns of transitions across Wave 1 (W1), Wave 2 (W2) and Wave 3 (W3) of the PATH Study. Taylor presents data on transitions among current users of cigarettes, ${ }^{5}$ Stanton presents data on transitions among current users of ENDS, ${ }^{6}$ Sharma presents data on transitions among current users of hookah, ${ }^{7}$ Edwards presents data on transitions among current users of cigars ${ }^{8}$ and Sharma presents data on transitions among current users of smokeless tobacco products. ${ }^{9}$ Additionally, Stanton presents data on transitions among never users of tobacco products. ${ }^{4}$ These papers also address whether tobacco use refers to one 
Table 1 Patterns of tobacco (product) use/non-use occurring over multiple time points

\begin{tabular}{lll}
\hline User state & Pattern & Definition \\
\hline $\begin{array}{l}\text { Current/former } \\
\text { users }\end{array}$ & Persistent use & Use across all time points \\
& Discontinued use & Use to no use \\
& Relapse/reuptake of use & Use, to no use, to use \\
& Transition to PTU & Exclusive use to PTU \\
& Transition to exclusive use & PTU to exclusive use \\
& Switching & Exclusive use to different product use \\
& Inconsistent PTU & PTU, to no PTU, to PTU \\
\hline Never users & Persistent never use & Never use across all time points \\
& Initiate use & Never use to new use
\end{tabular}

Definitions may include multiple patterns across time points, for example, discontinued use includes the use, to no use, to no use pattern, as well as the use, to use, to no use pattern; user states and patterns of use can refer to various frequencies of use, for example, current use can refer to past 30-day use or daily use, new use can refer to experimental use or regular use.

PTU, polytobacco use.

type of tobacco product (exclusive tobacco product use) or to two or more types of tobacco products (polytobacco use), which allows for more complex transition patterns to be considered, as introduced in table 1 and described in detail in each of these six papers. ${ }^{4-9}$

For each of these product-specific papers, ${ }^{4-9}$ we consider whether polytobacco use includes cigarette use because of the prevalence and harmfulness of cigarette use. ${ }^{25}$ In each of these papers, we highlight patterns of transitions based on supporting supplemental material that provides information on a large array of mutually exclusive and exhaustive categories at each time point that the research community can use as a resource, such as to inform future analyses on other transitions and trajectories.

In addition to the importance of describing tobacco use patterns longitudinally, it is equally important to understand what drives transition behaviours, particularly factors that may be modifiable or useful for targeting interventions. Figure 1 shows that the broad transitions of initiation, cessation and relapse can occur in steps (denoted by showing two arrows between user states); for example, cigarette smoking cessation is composed of moving from being a current smoker to making a quit attempt to succeeding in a quit attempt. Three papers in this issue describe correlates of the broad transitions of initiation, ${ }^{22}$ cessation $^{23}$ and relapse ${ }^{24}$ for each type of tobacco product, along with correlates of steps within each of these transitions over a 1-year time frame using data from W1 to W2 and from W2 to W3 of the PATH Study. Table 2 lists the individual-level initial correlates considered in this issue for each of the broad transitions and steps within them, though other factors at the environment level or product level, for example, may also relate to transition behaviours or interact with individual-level correlates to affect transition behaviours.

As with the papers in this issue that report on patterns of transitions over three time points, ${ }^{4-9}$ the papers focused on correlates $^{22-24}$ can also serve as building blocks for future papers. They provide only an initial look at some of the demographic and tobacco use factors that may influence tobacco use transitions.

\section{THE PATH STUDY}

The PATH Study is a nationally representative longitudinal tobacco use study of about 46000 Americans aged 12 years and older. In 2011, the National Institute on Drug Abuse, National Institutes of Health and the Center for Tobacco Products (CTP) at FDA launched the PATH Study to generate longitudinal epidemiological data on tobacco use behaviours among the US population of youth and adults. ${ }^{26}$ Data were collected from 12 September 2013 through 14 December 2014 (W1), from 23 October 2014 through 30 October 2015 (W2) and from 19 October 2015 through 23 October 2016 (W3), using audio computer-assisted self-interviews administered in English or Spanish, with future waves of data collection planned through 2024. At W1, the weighted response rate for the household screener was $54.0 \%$, and the overall weighted response rate was $74.0 \%$ for the adult interview and $78.4 \%$ for the youth interview. At W2, the overall weighted response rate was $83.2 \%$ for the adult interview and $87.3 \%$ for the youth interview, and at W3 it was $78.4 \%$ for the adult interview and $83.3 \%$ for the youth interview. Further details regarding the PATH Study design and W1 methods are published elsewhere. ${ }^{26}$ Details on interviewing procedures, questionnaires, sampling, weighting, response rates and accessing the data are described in the PATH Study Restricted Use Files User Guide on https://doi.org/10.3886/Series606. ${ }^{27} \mathrm{The}$ Study was conducted by Westat and approved by the Westat Institutional Review Board. All respondents ages 18 and older provided informed consent, with youth respondents ages 12-17 providing assent while each one's parent/legal guardian provided consent.

\section{THE PATH STUDY IN THE CONTEXT OF OTHER LARGE-SCALE TOBACCO DATA COLLECTION SYSTEMS IN THE USA AND INTERNATIONALLY}

While the PATH Study is a nationally representative US study designed to inform FDA's tobacco regulatory activities, it has design features that complement other large-scale tobacco data collection systems. Differentiating features of the PATH Study include: (1) longitudinal design for both tobacco users and non-users; (2) depth and breadth of information about a wide array of tobacco products; and (3) collection of biospecimens

Table 2 Correlates of broad transition behaviours

\begin{tabular}{|c|c|c|c|c|}
\hline \multicolumn{3}{|c|}{ Transitions } & \multirow{2}{*}{$\begin{array}{l}\text { Correlates } \\
\text { Age group }\end{array}$} & \multirow{2}{*}{$\begin{array}{l}\text { Categories } \\
12-14,15-17,18-24,25-39,40-54,55+\text { years }\end{array}$} \\
\hline \multirow[t]{9}{*}{ Initiation } & Cessation & Relapse & & \\
\hline & & & Sex & Male, female \\
\hline & & & Sexual orientation & Straight, gay/lesbian, bisexual, something else (ages 14+ years) \\
\hline & & & Race/ethnicity & Non-Hispanic white, non-Hispanic black/African-American, non-Hispanic other, Hispanic \\
\hline & & & Educational attainment & Less than high school (HS), HS graduate, some college/Associate's degree, Bachelor's degree+ (adults) \\
\hline & & & Household income & $<\$ 25000, \$ 25000-\$ 74999, \geq \$ 75000$, not reported (adults) \\
\hline & & & Other tobacco product use & Use/no use of other tobacco products \\
\hline & & & Dependence & Overall tobacco dependence score \\
\hline & & & Time since last use & Less than 1 year, $1-2$ years, $2+$ years \\
\hline
\end{tabular}


longitudinally in addition to questionnaire data. Below is a brief description of selected international and US tobacco surveys and studies designed to be representative of certain geographic areas.

Internationally, the Global Adult Tobacco Survey ${ }^{28}$ and the Global Youth Tobacco Survey ${ }^{29}$ are standards for systematically monitoring and tracking tobacco use and tobacco control indicators cross-sectionally using a standard protocol, as part of the global effort to reduce tobacco smoking outlined in the WHO FCTC. ${ }^{13}$ These surveys are intended to create nationally representative estimates for each country, but also to allow data between countries to be compared.

The International Tobacco Control Policy Evaluation Project ${ }^{30}$ collects longitudinal data in $25+$ countries primarily from tobacco users with a focus on policy evaluation guided by WHO FCTC domains. A similar survey design is used in each country for the primary purpose of evaluating proximal and distal policy effects by comparing both pre/postintervention in a given country as well as to other comparison countries where no such policy was implemented.

In the USA, many national data systems exist but most are cross-sectional, not designed to assess tobacco policies or regulations, or do not collect biospecimens. Data systems like the National Health Interview Survey, ${ }^{31}$ National Survey on Drug Use and Health ${ }^{32}$ and the Tobacco Use Supplement to the Current Population Survey (TUS-CPS) ${ }^{33}$ provide national-level estimates of tobacco use for some products, and TUS-CPS allows for state-specific estimates.

The National Youth Tobacco Survey ${ }^{34}$ and the previously conducted National Adult Tobacco Survey ${ }^{35}$ are cross-sectional and provide national and state data on tobacco use to evaluate comprehensive tobacco control programme activities. The Monitoring the Future ${ }^{36}$ survey is a cross-sectional school-based survey like the National Youth Tobacco Survey and allows for national estimates with a particular focus on drug use behaviours. The National Health and Nutrition Examination Survey ${ }^{37}$ is a nationally representative cross-sectional in-person survey that includes a very detailed health assessment, physician examination and biomarkers, though it has a relatively limited assessment of tobacco use behaviours. The Behavioral Risk Factor Surveillance System uses telephone surveys to collect data at the state and local levels regarding health-related risk behaviours, chronic health conditions and use of preventive services, though it has relatively limited tobacco use assessments. ${ }^{38}$

The PATH Study blends many of the desirable design features of these studies to serve its role in informing the regulatory activities of FDA's CTP. While the PATH Study is designed for this regulatory purpose, the data are rich and informative to tobacco control investigators internationally to better understand what products people are using, why and to what effect.

\section{HOW CAN THE DATA FROM THE PATH STUDY BE USED BY THE RESEARCH COMMUNITY?}

The PATH Study is relevant and important to a broad international audience in the following three areas: (1) the role of FDA tobacco regulation in areas that have international relevance; (2) the larger area of tobacco policy research; and (3) methods and epidemiological research. The PATH Study is designed with FDA tobacco regulation needs in mind. Results from the PATH Study could provide scientific evidence for FDA regulatory actions, such as rulemaking and estimating the potential effect of regulations over time, or for help with public education campaigns, for example. Work from the PATH Study before and after the sale of new tobacco products is permitted in the USA or tobacco product design standards are implemented, for example, can benefit those in other countries with or considering similar policies.

The PATH Study can also be used to assess tobacco policies such as restrictions on tobacco product advertising ${ }^{39}$ or home smoking policies. ${ }^{40}$ In addition, data on the baseline state of residence of PATH Study participants can be linked to other policy-relevant data, such as tobacco price data, to better understand how these policies outside of FDA's regulatory scope impact behaviour.

Furthermore, as a large-scale longitudinal study, there is a tremendous amount of information that can be used for new measures development and epidemiological studies with far-reaching relevance. For example, measures of dependence have been validated for different tobacco products. ${ }^{41}$ Further, PATH Study analyses of tobacco use in specific subpopulations such as sexual, gender or racial/ethnic minorities, those who use illicit substances, or those with mental health disorders can further our understanding of how and why tobacco use evolves in these populations.

\section{FUTURE DIRECTIONS}

The conceptual overview presented here can be expanded, and additional empirical work can build on the data presented in the papers in this issue, incorporating more details that are important to understanding tobacco use behaviours. For example, measures of tobacco product use frequency, intensity, as well as biomarkers of harm can be added to better understand how behaviour is related to health. Furthermore, additional correlates can be examined, particularly in the context of natural experiments where some policy or intervention is adopted in some place(s) but not others, to better understand what factors drive observed tobacco use patterns. For example, state-identifier data can be used to study the impact on tobacco transition behaviours of state-level restrictions on the sale of menthol-flavoured tobacco products, although geocoding of data for assessment of local policies is not available to protect respondent confidentiality. As additional waves of data are collected, the PATH Study can provide increasingly powered, detailed data to estimate parameters. The PATH Study data are available to the research community through the National Addiction and HIV Data Archive Program (https://doi.org/10. $3886 /$ Series606) $)^{27}$ and we encourage investigators to use the data to address tobacco research questions.

\section{What this paper adds}

Models have been developed to estimate the population health impacts of tobacco initiation, cessation, and relapse transitions.

- Models should be informed by high quality, longitudinal data to estimate these constructs.

- We provide a high-level conceptual overview for understanding tobacco transition behaviours and correlates of these behaviours using data from the Population Assessment of Tobacco and Health (PATH) Study, a U.S. nationally representative longitudinal tobacco study of about 46,000 persons aged $12+$ years.

Contributors All authors contributed to the conceptual design of the work. AH and KAK drafted the work and all authors critically revised it. All authors approved the work for journal publication and agree to be accountable for all aspects of the work.

Funding This manuscript is supported with Federal funds from the National Institute on Drug Abuse, National Institutes of Health, and the Center for Tobacco Products, Food and Drug Administration, Department of Health and Human Services, under contract to Westat (Contract No. HHSN271201100027C). 
Disclaimer The findings and conclusions in this report are those of the authors and do not necessarily represent the official position of the U.S. Department of Health and Human Services or any of its affiliated institutions or agencies.

Competing interests WMC reports long-term stock holdings in General Electric Company, 3M Company, and Pfizer Incorporated, unrelated to this manuscript. No financial disclosures were reported by the other authors of this paper.

Patient consent for publication Not required.

Ethics approval The PATH Study was conducted by Westat and approved by the Westat Institutional Review Board. All respondents ages 18 and older provided informed consent, with youth respondents ages 12-17 providing assent while each one's parent/legal guardian provided consent.

Provenance and peer review Not commissioned; externally peer reviewed.

Data availability statement There are no data in this work

\section{ORCID iDs}

Karin A Kasza http://orcid.org/0000-0002-4492-098X

Heather L Kimmel http://orcid.org/0000-0001-8278-0095

Kristie A Taylor http://orcid.org/0000-0001-6668-7923

Eva Sharma http://orcid.org/0000-0002-1328-508X

Kathryn C Edwards http://orcid.org/0000-0002-0645-1705

Michael J Halenar http://orcid.org/0000-0002-8703-3811

Maansi Bansal-Travers http://orcid.org/0000-0002-7944-3570

Lisa D Gardner http://orcid.org/0000-0003-3340-2818

Cassandra A Stanton http://orcid.org/0000-0001-5329-6261

\section{REFERENCES}

1 Asma S, Mackay J, Song SY, et al. The GATS atlas. Atlanta, GA: CDC Foundation, 2015. http://www.gatsatlas.org/

2 Gravely S, Fong GT, Cummings KM, et al. Awareness, trial, and current use of electronic cigarettes in 10 countries: findings from the ITC project. Int I Environ Res Public Health 2014;11:11691-704.

3 Kasza KA, Ambrose BK, Conway KP, et al. Tobacco-Product use by adults and youths in the United States in 2013 and 2014. N Engl J Med 2017;376:342-53.

4 Stanton CA, Sharma E, Seaman E, et al. Initiation of any tobacco and five tobacco products across 3 years among youth, young adults, and adults in the USA: findings from the PATH Study Waves 1-3 (2013-2016). Tob Control 2020;29:s178-90.

5 Taylor KA, Sharma E, Edwards KC, et al. Longitudinal pathways of exclusive and polytobacco cigarette use among youth, young adults, and adults in the USA: findings from the PATH Study Waves 1-3 (2013-2016). Tob Control 2020:29:5139-46.

6 Stanton CA, Sharma E, Edwards KC, et al. Longitudinal transitions of exclusive and polytobacco electronic nicotine delivery systems (ends) use among youth, young adults, and adults in the USA: findings from the PATH Study Waves 1-3 (2013-2016). Tob Control 2020;29:s147-54.

7 Sharma E, Bansal-Travers M, Edwards KC, et al. Longitudinal transitions of exclusive and polytobacco hookah use among youth, young adults, and adults in the USA: findings from the PATH Study Waves 1-3 (2013-2016). Tob Control 2020;29:s155-62.

8 Edwards KC, Sharma E, Halenar MJ, et al. Longitudinal pathways of exclusive and polytobacco cigar use among youth, young adults, and adults in the USA: findings from the PATH Study Waves 1-3 (2013-2016). Tob Control 2020;29:5163-9.

9 Sharma E, Edwards KC, Halenar MJ, et al. Longitudinal pathways of exclusive and polytobacco smokeless use among youth, young adults, and adults in the USA: findings from the PATH Study Waves 1-3 (2013-2016). Tob Control 2020;29:s170-7.

10 National Center for Chronic Disease Prevention and Health Promotion (US) Office on Smoking and Health. The health consequences of Smoking - 50 years of progress: a Report of the Surgeon General. Atlanta, GA: Centers for Disease Control and Prevention (US), 2014.

11 Jha P, Ramasundarahettige C, Landsman V, et al. 21St-Century hazards of smoking and benefits of cessation in the United States. N Engl J Med 2013:368:341-50.

12 U.S. Department of Health and Human Services. The health benefits of smoking cessation. U.S. Department of Health and Human Services. Public Health Service. Centers for Disease Control. Center for Chronic Disease Prevention and Health Promotion. Office on Smoking and Health, 1990.

13 WHO Framework Convention on Tobacco Control. Available: http://www.who.int/fctc/ en/

14 111th Congress. Family Smoking Prevention and Tobacco Control Act. H.R. 1256, 2009: 6-16.
15 Tobacco product standard for nicotine level of combusted cigarettes, 83 fed. reg. 11818 [Accessed 16 Mar 2018].

16 Feirman SP, Glasser AM, Rose S, et al. Computational models used to assess US tobacco control policies. Nicotine Tob Res 2017:19:1257-67.

17 Feirman SP, Donaldson E, Glasser AM, et al. Mathematical modeling in tobacco control research: initial results from a systematic review. NICTOB 2016;18:229-42.

18 Apelberg BJ, Feirman SP, Salazar E, et al. Potential public health effects of reducing nicotine levels in cigarettes in the United States. N Engl J Med 2018;378:1725-33.

19 Vugrin ED, Rostron BL, Verzi SJ, et al. Modeling the potential effects of new tobacco products and policies: a dynamic population model for multiple product use and harm. PLoS One 2015;10:e0121008.

20 Levy DT, Cummings KM, Villanti AC, et al. A framework for evaluating the public health impact of e-cigarettes and other vaporized nicotine products. Addiction 2017;112:8-17.

21 Cobb CO, Villanti AC, Graham AL, et al. Markov modeling to estimate the population impact of emerging tobacco products: a proof-of-concept study. Tob Regul Sci 2015;1:129-41.

22 Kasza KA, Edwards KC, Tang Z, et al. Correlates of tobacco product initiation among youth and adults in the USA: findings from the PATH Study Waves 1-3 (2013-2016). Tob Control 2020;29:s191-202.

23 Kasza KA, Edwards KC, Tang Z, et al. Correlates of tobacco product cessation among youth and adults in the USA findings from the PATH Study Waves 1-3 (2013-2016). Tob Control 2020;29:s203-15.

24 Edwards KC, Kasza KA, Tang Z, et al. Correlates of tobacco product reuptake and relapse among youth and adults in the United States: findings from the path study waves 1-3 (2013-2016). Tob Control 2020;29:s216-26.

25 U.S. Public Health Service. Smoking and Health: Report of the Advisory Committee to the Surgeon General of the Public Health Service. U.S. Department of Health, Education, and Welfare, Public Health Service, Center for Disease Control, 1964

26 Hyland A, Ambrose BK, Conway KP, et al. Design and methods of the Population Assessment of Tobacco and Health (PATH) Study. Tob Control 2017;26:371-8.

26 Population Assessment of Tobacco and Health (PATH) Study. Available: https://doi.org/ 10.3886/Series606

28 Palipudi KM, Morton J, Hsia J, et al. Methodology of the Global Adult Tobacco Survey 2008-2010. Glob Health Promot 2016;23:3-23.

29 Warren CW, Riley L, Asma S, et al. Tobacco use by youth: a surveillance report from the Global Youth Tobacco Survey project. Bull World Health Organ 2000;78:868-76.

30 International Tobacco Control Policy Evaluation Project (ITC Project). Available: https:// itcproject.org/

31 National Health Interview Survey (NHIS). Centers for Disease Control and Prevention. Atlanta, GA. Available: https://www.cdc.gov/nchs/nhis/index.htm

32 National Survey on Drug Use and Health (NSDUH). Substance Abuse and Mental Health Services Administration. Rockville, MD. Available: https://nsduhweb.rti.org/ respweb/homepage.cfm

33 Tobacco Use Supplement to the Current Population Survey (TUS-CPS). National Cancer Institute. Available: https://cancercontrol.cancer.gov/brp/tcrb/tus-cps/

34 National Youth Tobacco Survey (NYTS). Centers for Disease Control and Prevention. Atlanta, GA. Available: http://www.cdc.gov/tobacco/data_statistics/surveys/nyts/

35 National Adult Tobacco Survey (NATS). Centers for Disease Control and Prevention. Atlanta, GA. Available: https://www.cdc.gov/tobacco/data_statistics/surveys/nats/ index.htm

36 Monitoring the Future (MTF). University of Michigan. Ann Arbor. Available: http:// www.monitoringthefuture.org

37 National Health and Nutrition Examination Survey (NHANES). Centers for Disease Control and Prevention. Atlanta, GA. Available: https://www.cdc.gov/nchs/nhanes/ index.htm

38 Behavioral Risk Factor Surveillance System (BRFSS). Centers for Disease Control and Prevention. Atlanta, GA. Available: https://www.cdc.gov/brfss/about/index.htm

39 Pierce JP, Sargent JD, White MM, et al. Receptivity to tobacco advertising and susceptibility to tobacco products. Pediatrics 2017;139:e20163353.

40 Hyland B, Hyland A, Rivard C, et al. Tobacco-Free home policies: longitudinal findings from Wave 1 (2013-14) and Wave 2 (2014-15) of the Population Assessment of Tobacco and Health (PATH) Study. Annual Meeting of the Society for Research on Nicotine and Tobacco, 2018

41 Strong DR, Pearson J, Ehlke S, et al. Indicators of dependence for different types of tobacco product users: descriptive findings from Wave 1 (2013-2014) of the Population Assessment of Tobacco and Health (PATH) Study. Drug Alcohol Depend 2017;178:257-66 